\title{
Cultura Organizacional y Capacidad de Ejecución
}

\section{Maximiliano E. Jiménez Arbeláez ${ }^{1 *}$.}

${ }^{1}$ Presidente de MJ Consulting, Inc. Obarrio, Ave. Samuel Lewis Torre ADR Piso 7 Panamá. Profesor y Decano, Facultad de Negocios, Universidad Católica Sata María la Antigua (USMA) Panamá, República de Panamá.

*Autor para correspondencia. Email: maxejimenez@gmail.com

Recibido: 22 de febrero de 2018

Aceptado: 18 de abril de 2018

\section{Introducción}

Durante la última década, el tema de la ejecución de la estrategia ha tomado gran interés en los negocios. Este interés se refleja en la publicación de varios libros dedicados al tema, entre ellos Execution: The Discipline to Get Things Done (Bossidy y Charan 2002), Making Strategy Work:

Leading Effective Execution and Change (Hrebiniak 2005) y The Execution Premium: Linking Strategy to Operations for Competitive Advantage (Kaplan y Norton 2008). Una variedad de estudios recientes respaldan el interés en la ejecución. Por ejemplo, en una encuesta de la American Management Association del 2006, sólo el 3\% respondió que sus empresas eran muy exitosas en la ejecución de la estrategia, mientras que el $62 \%$ calificó esta capacidad como mediocre o peor. Otra encuestas del Conference Board en 2007 y del Monitor Group en 2006 señalan que la prioridad número uno de los ejecutivos es la excelencia en la ejecución. Finalmente, en su encuesta internacional de 2008, la Society for Human Resource Management indica que las prioridades 1 y 2 de los ejecutivos encuestados eran el desarrollo organizacional y la gestión estratégica, respectivamente, ambos temas íntimamente relacionados con la ejecución de la estrategia.

Estos resultados no son una sorpresa. Varias tendencias en el entorno de los negocios se conjugan para dificultar la ejecución de la estrategia: la creciente complejidad de los negocios, la inestabilidad del entorno y la dificultad para predecir el futuro, y el aumento exponencial de la competencia. El denominador común de estas tendencias es el cambio rápido y el principal inductor del mismo es el avance de la tecnología que trasforma modelos de negocios y elimina las barreras entre unidades de negocios, empresas, sectores, países y regiones.

La práctica y las investigaciones demuestran que el desempeño de la empresa se optimiza cuando su estrategia responde a las exigencias del entorno y cuando su diseño organizacional facilita la ejecución de la estrategia. Ambas la estrategia y el diseño organizacional son esenciales. Frente a un entorno de cambios turbulentos, las competencias organizacionales de flexibilidad, adaptación e innovación son indispensables para la competitividad y el desarrollo empresarial sostenible. 
Invest. pens. crit. (ISSN 1812-3864)

Vol. 6, No. 1, enero-abril 2018

pp. $65-80$

El propósito de este artículo es presentar un marco de referencia para entender y mejorar desempeño de las empresas desde la perspectiva del diseño organizacional, enfatizando el rol de la cultura organizacional. Para ello, divido el artículo en cuatro partes que siguen a esta introducción. La primera parte presenta un modelo del sistema organizacional para fines de diagnóstico y diseño e incluye un repaso de los resultados de investigaciones recientes sobre prácticas gerenciales conducentes al alto rendimiento, destacando el rol de la cultura organizacional.

La segunda parte integra los hallazgos referidos en la primera parte dentro del concepto de las organizaciones de alto rendimiento. Frente a este marco, la tercera parte presenta los resultados de mediciones de la cultura organizacional realizadas por el autor en diez empresas panameñas importantes y ofrece una posible explicación de los resultados obtenidos haciendo referencia a la cultura nacional. Esta parte termina con una discusión de las implicaciones de los hallazgos en relación a la competitividad y la sostenibilidad del alto rendimiento. La cuarta y última parte ofrece nuestras conclusiones y recomendaciones para lograr una mayor capacidad de ejecución e innovación en las empresas panameñas.

\section{Ejecución de la Estrategia y Cultura Organizacional}

La estrategia se ejecuta a través del sistema organizacional. Existen varios modelos útiles para entender en forma integral los componentes del sistema organizacional y facilitar su diagnóstico y diseño. Ejemplos de estos modelos son el modelo de los requerimientos para el procesamiento de la información de Nadler y Tushman (1997), el modelo de la estrella (estrategia, estructura, procesos laterales, sistemas de recompensas y prácticas de recursos humanos) de Galbraith (1995) y el modelo de las Siete S propuesto primeramente por Pacale y Athos (1981) y popularizado por Peters y Waterman en el best seller "En Busca de la Excelencia" (1982) y luego por la firma McKinsey \& Co.

El modelo de las Siete S (s por la primera letra en inglés de cada uno de sus siete componentes) argumenta que existen siete dimensiones básicas que representan las actividades gerenciales medulares. Estas siete dimensiones o componentes del sistema organizacional son: estrategia (dirección y ventaja competitiva), estructura (relaciones de autoridad y responsabilidad), sistemas (procesos gerenciales y procesos de negocios), estilos (comportamientos y relaciones interpersonales de los gerentes), gente (competencias y actitudes del personal), competencias corporativas, y valores compartidos (cultura).

En mi práctica profesional uso un modelo de siete componentes interdependientes similar al de las Siete S, cuyos componentes aparecen en la Figura 1 y paso a describir a continuación: 
Invest. pens. crit. (ISSN 1812-3864)

Vol. 6, No. 1, enero-abril 2018

pp. $65-80$

Figura 1. Componentes del Sistema Organizacional

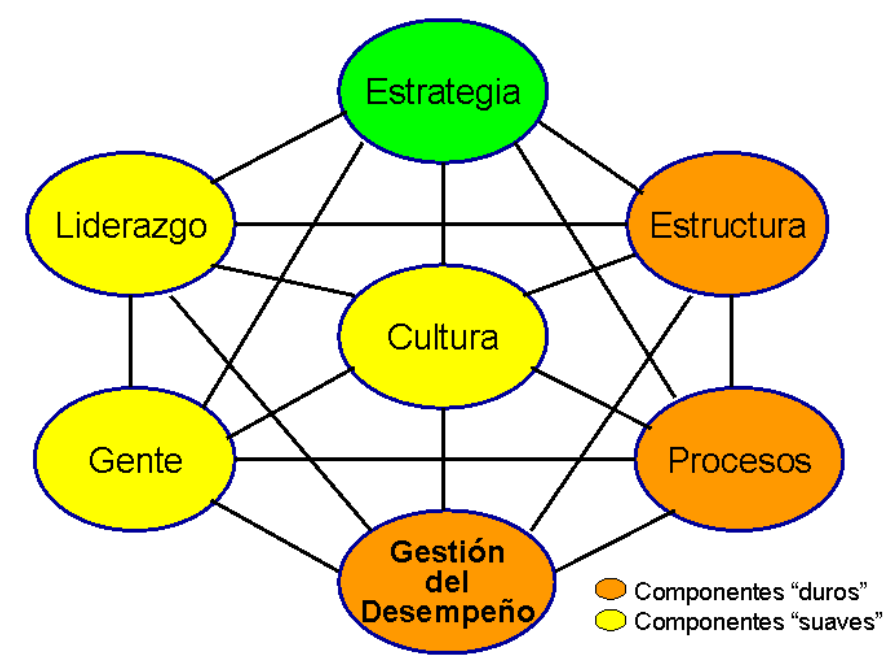

$\checkmark$ Estrategia: Selección de clientes, productos y actividades en la entrega de una proposición de valor ganadora a los stakeholders - la forma de cómo se intenta crear valor de manera sostenible.

$\checkmark$ Estructura: Agrupación de funciones y distribución de la autoridad y responsabilidad en la ejecución de las tareas, y los mecanismos laterales de coordinación.

$\checkmark$ Procesos: Procesos gerenciales (toma de decisiones, planeación, asignación de recursos, identificación y resolución de problemas) y procesos de negocios (actividades realizadas para la entrega de valor a los clientes).

$\checkmark$ Gestión del desempeño: Sistemas de objetivos, indicadores de desempeño claves (KPIs) y scorecards; prácticas de seguimiento, evaluación, retroalimentación y mejora del desempeño; y sistemas de recompensas.

$\checkmark$ Gente: Competencias y motivación del personal, y prácticas para la atracción, desarrollo y retención del talento humano y la creación de un contexto del trabajo conducente al alto rendimiento.

$\checkmark$ Liderazgo: Estilo de liderazgo y valores demostrados en conductas, efectividad de la alta gerencia como equipo, y competencias gerenciales, interpersonales y funcionales del grupo de gerencia.

$\checkmark$ Cultura: Creencias, expectativas, normas de conducta y valores compartidos que se reflejan en los patrones de conducta de la gente en la organización. 
Invest. pens. crit. (ISSN 1812-3864)

Vol. 6, No. 1, enero-abril 2018

pp. $65-80$

Este modelo ofrece una herramienta robusta para el diagnóstico y diseño de una plataforma organizacional que responda a las características del negocio, cumpla con los requisitos de la estrategia y se adapte ventajosamente a los cambios del entorno de los negocios. Específicamente, el sistema organizacional tiene como objetivo central generar las capacidades organizacionales (competencias, procesos, aprendizaje, tecnologías, etc.) requeridas para ejecutar la estrategia. Así, el diseño organizacional es un proceso que busca configurar los diferentes componentes del sistema organizacional para que en forma coherente y alineada desarrollen las competencias necesarias para ejecutar la estrategia.

Como puede inferirse del diagrama presentado, además de mostrar interdependencia, la ubicación de los componentes no es casual. Por ejemplo, el liderazgo se ubica frente a la estructura (organigrama), la estrategia frente a los sistemas de gestión (seguimiento cuantitativo de la ejecución de la estrategia) y la gente frente a los procesos. Dentro de este esquema nos interesa resaltar el rol de la cultura.

La cultura organizacional pasa de ser un tema académico a un tema de interés en el ámbito de los negocios a principios de los ochenta, principalmente gracias al libro "En Busca de la Excelencia". Así comienza a considerarse a la cultura como uno de los elementos claves en el desempeño empresarial y a los líderes como los forjadores primordiales de la cultura organizacional (Schein 2004, Hall 1988).

Básicamente, la importancia de la cultura radica en que es a través de las personas que se realizan las tareas de la organización. Más puntualmente, es a través de los comportamientos de las personas, mediatizados por sus actitudes, valores y creencias sobre la gente que las personas realizan dichas tareas. En este sentido, puede considerarse a la cultura como un poderoso sistema que influye sobre el comportamiento de las personas. Y estos comportamientos pueden promover u obstaculizar el logro de los objetivos de la organización y afectar su postura hacia el cambio, la innovación, las relaciones interpersonales y, en general, influir sobre todas las actividades que realiza la empresa. De allí su ubicación en el centro del sistema organizacional.

Aparte de estas consideraciones "cualitativas" ¿es importante la cultura desde el punto de vista del desempeño económico a largo plazo? Los profesores Kotter y Heskett (1992) de la Universidad de Harvard realizaron posiblemente el primer estudio sistemático a largo plazo de la relación entre la cultura y el desempeño económico corporativo. Ellos investigaron durante un periodo de 11 años 207 empresas en los EUA, comparando los rasgos de sus culturas organizacionales con los resultados financieros. Sus hallazgos destacan la adaptabilidad de la empresa como una competencia organizacional crítica e identifican dos rasgos culturales ligados al alto desempeño: (1) el alineamiento de la atención y esfuerzos hacia los stakeholders claves (clientes, empleados y accionistas) y (2) el desarrollo de liderazgo a todos los niveles de la organización. A continuación los resultados comparativos durante el periodo estudiado de las empresas que mostraron los dos rasgos de cultura identificados vs. el resto de las empresas:

$\checkmark$ En el periodo de estudio las ventas crecieron un $682 \%$ vs. $116 \%$.

$\checkmark$ La fuerza laboral aumentó un $282 \%$ vs. $36 \%$

$\checkmark$ El precio de las acciones aumentó un $901 \%$ vs. $74 \%$

$\checkmark$ La utilidad neta aumentó un $756 \%$ vs. $1 \%$ 
Invest. pens. crit. (ISSN 1812-3864)

Vol. 6, No. 1, enero-abril 2018

pp. $65-80$

Estos resultados no dejan duda sobre la importancia de la cultura sobre el desempeño empresarial.

Otro estudio más reciente de los profesores Nohria, Joyce y Roberson (2003) demuestra igualmente la importancia "pragmática" de los temas ligados a la cultura. Su investigación abarcó el estudio detallado de 160 empresas en los EUA durante 10 años (1986-1996) y la utilización por estas empresas de cerca de 200 prácticas gerenciales reconocidas. La investigación buscaba contestar dos preguntas: ¿Por qué algunas empresas consistentemente sobrepasan a sus competidores? y ¿Cuáles de cientos de herramientas y técnicas gerenciales bien establecidas pueden ayudar a una empresa a ser altamente exitosa?

La diferencia en el desempeño financiero entre las empresas consistentemente superiores y las no exitosas fue abrumadora. Así, el grupo de empresas ganadoras demostró como portafolio, un retorno de $\$ 11$ por cada $\$ 1$ invertido durante los 10 años estudiados, mientras que el retorno en las otras empresas estudiadas fue de sólo $\$ 1.50$.

Como resultado de su investigación, los autores demostraron que de las casi 200 prácticas gerenciales identificadas, sólo cuatro, manejadas excelentemente, fueron comunes en todas las empresas ganadoras. Adicionalmente, las empresas exitosas complementaron estas prácticas primarias con un manejo superior en dos prácticas gerenciales secundarias. A este hallazgo le llamaron la fórmula $4+2$ para el éxito de los negocios.

Las cuatro prácticas gerenciales primarias, aquellas manejadas excelentemente por todas las empresas ganadoras son:

1) Estrategia: Lo importante no es tanto la naturaleza de la estrategia sino que la misma sea comunicada con claridad, entendida y apoyada por toda la organización, y comunicada coherentemente a los demás stakeholders. En las empresas ganadoras la gente sabe cómo su trabajo contribuye al éxito de la empresa.

2) Ejecución: Al igual que con la estrategia, lo importante no es el qué sino el cómo. Por ejemplo, no se encontró correlación significativa entre la utilización de herramientas reconocidas (ERP, CRM, terciarización, etc.) y el desempeño financiero. Lo que hacía la diferencia es una atención disciplinada a la ejecución eficiente y productiva de las operaciones.

3) Cultura: Todavía muchas empresas consideran la cultura como un tema soft al cual no se le presta mucha atención. En otras empresas se le toma en serio, pero el énfasis es en lograr ambientes "divertidos" asumiendo que esto conduce a la lealtad y a la productividad del personal. Los hallazgos de esta investigación demuestran que promover un ambiente donde la gente disfrute del trabajo no es ni cercanamente tan importante como desarrollar una cultura que enfatice el alto rendimiento, la rendición de cuentas (accountability) y un comportamiento ético. Estas culturas promueven la contribución tanto de individuos como de grupos, y hacen que todos en la organización se sientan responsables por los resultados y el éxito de la empresa.

4) Estructura: Ningún tipo de estructura organizacional pudo diferenciar consistentemente los ganadores de los perdedores. Lo decisivo fue contar con organizaciones sencillas, rápidas, 
Invest. pens. crit. (ISSN 1812-3864)

Vol. 6, No. 1, enero-abril 2018

pp. $65-80$

flexibles y achatadas. Las organizaciones demasiado burocráticas tendían a obstaculizar el cambio y desanimaban a los empleados. La clave del éxito resultó ser contar con estructuras que simplifiquen el trabajo, faciliten la coordinación y promuevan la agilidad organizacional.

En adición a un manejo superior de las prácticas gerenciales primarias, las empresas ganadoras demostraron excelencia en dos de cuatro prácticas gerenciales secundarias. Fue interesante observar que un excelente manejo en más de dos de estas prácticas no ofreció beneficios significativos. A continuación las prácticas gerenciales secundarias.

1) Manejo del talento: Atraer, desarrollar y retener talento. De acuerdo a los investigadores el mejor signo de un excelente manejo del talento fue la facilidad con la que una empresa reemplazaba a un ejecutivo saliente con otro de adentro. De hecho, los ganadores contrataron presidentes ejecutivos de afuera la mitad de las veces que los perdedores. Aproximadamente un $50 \%$ de las empresas ganadoras demostraron excelencia en esta práctica, dedicando importantes recursos para desarrollar y retener su personal.

2) Innovación: La empresas exitosas que mostraron un manejo superior en la innovación se caracterizaron por una ambición de liderar las transformaciones innovadoras dentro del sector industrial al que pertenecían. Estas empresas no escatimaron en "canibalizar" productos existentes para impulsar nuevos productos. No hubo predominio de ninguna de las fuentes de innovación generalmente aceptadas ( $\mathrm{I}+\mathrm{D}$, laboratorios externos, alta gerencia, empleados, clientes y proveedores) en las empresas innovadoras. Fundamentalmente, estas empresas demostraron una "cultura de innovación" cuyos rasgos incluyen la orientación hacia la mejora continua, la tolerancia al riesgo y la atención a los cambios disruptivos en tecnologías y el mercado. Menos de la mitad de las empresas ganadoras demostró excelencia en esta práctica secundaria lo cual refleja la dificultad de un manejo superior de la innovación.

3) Liderazgo: De todas las características asociadas con un liderazgo eficaz, las siguientes probaron ser comunes en los líderes de las empresas ganadoras que demostraron excelencia en esta práctica: (1) la habilidad para desarrollar relaciones con la gente a todos los niveles de la organización y para inspirar al resto del equipo gerencial a hacer lo mismo, y (2) la habilidad para detectar oportunidades y problemas tempranamente y de actuar rápidamente para aprovechar las oportunidades o resolver los problemas.

4) Fusiones y alianzas: Las fusiones y alianzas son una importante fuente de crecimiento. Aunque muchas de las empresas estudiadas realizó adquisiciones durante el periodo de estudio, solamente un $22 \%$ demostró un manejo eficaz de esta práctica. Los resultados demostraron que aquellas empresas que realizan adquisiciones relativamente pequeñas en forma consistente (dos o tres por año) tienden a ser más exitosas que aquellas que realizan grandes adquisiciones ocasionalmente. Por otro lado, ninguna de las empresas ganadoras realizó adquisiciones alejadas a su negocio central.

Cabe destacar que un análisis detallado de las prácticas primarias y secundarias identificadas por Nohria et al., con la excepción de las fusiones y alianzas, revelaría que subyacente a dichas prácticas existe una cultura de confianza en la gente y en su capacidad de realizar un excelente trabajo siempre y cuando 
Invest. pens. crit. (ISSN 1812-3864)

Vol. 6, No. 1, enero-abril 2018

pp. $65-80$

cuente con las competencias, las herramientas y la motivación apropiadas para realizarlo. Este rasgo fundamental de la cultura sería clave para la capacidad de ejecución de la estrategia.

En este sentido, Neilson et al. (2008) de la firma Booz and Co. realizaron una encuesta donde participaron más de mil empresas, con fines y sin fines de lucro, en 50 países. El objetivo de esta investigación fue identificar las características más determinantes para una ejecución exitosa de la estrategia. En una clasificación indexada basada en 100 puntos, la característica más importante fue "todos tienen una idea clara de las decisiones y acciones por las que son responsables". En organizaciones con alta capacidad de ejecución, $71 \%$ de las personas estuvieron de acuerdo con esta afirmación, mientras que este porcentaje descendió a 32\% cuando los encuestados provenían de organizaciones con baja capacidad de ejecución.

Los autores clasificaron las características vinculadas a la capacidad de ejecución en cuatro grupos: derechos sobre las decisiones (claridad en cuanto a quién es responsable por cuál decisión), información (disponibilidad, relevancia, uso de métricas), motivadores (evaluación del desempeño y bonificaciones) y estructura (tamaño de las unidades organizacionales y frecuencia y prácticas de promociones laterales y verticales). A continuación los resultados globales en orden de importancia según el índice de fortaleza de la característica:
$\checkmark$ Información: 54
$\checkmark$ Derechos de decisión: 50
$\checkmark$ Motivadores: 26
$\checkmark$ Estructura: 25

El Dr. Roger Martin (2010) de la Universidad de Toronto, Canadá, presenta otro estudio revelador sobre la capacidad de ejecución. En su artículo The Execution Trap, él argumenta que trazar una línea divisoria entre la formulación de la estrategia y la ejecución es casi garantía de fracaso. La práctica extendida de que la alta gerencia es responsable por la formulación de la estrategia y que el resto de la organización es responsable de la ejecución es un grave error. El, como otros lo han hecho (léase, por ejemplo, Kaplan y Norton, 2001) afirma que la estrategia debe ser el trabajo de todos en la organización. Cada persona actúa estratégicamente cuando su conducta está alineada con la estrategia. Para ello, durante la formulación de la estrategia deben utilizarse mecanismos de participación que abonen el camino para una ejecución eficaz.

El Dr. Martin respalda sus conclusiones con los resultados reportados por una encuesta realizada en marzo de 2010 al consejo de asesores de la revista Harvard Business Review, un grupo de opinión de lectores de la revista (1075 respondieron la encuesta). Así, por ejemplo, el 43\% de los encuestados no podían describir completamente la estrategia de sus empresas y la situación era mucho peor entre aquellos no involucrados en la formulación de la estrategia. Por otro lado, la percepción sobre la capacidad de formular una buena estrategia varió dependiendo de la participación en la formulación: $48 \%$ de los que participaban en la formulación creía que la organización era buena creando estrategias; este porcentaje descendió a 33\% entre los que no participaban en la formulación de la estrategia. Los encuestados señalaron que el aspecto más importante de la ejecución era la clarificación de la estrategia a toda la organización y que el mayor obstáculo a la ejecución era la falta de una traducción de la 
Invest. pens. crit. (ISSN 1812-3864)

Vol. 6, No. 1, enero-abril 2018

pp. $65-80$

estrategia al lenguaje de las operaciones, que tuviera sentido para el personal de línea y que ayudara a alinear el trabajo realizado con la estrategia.

Es interesante observar que los resultados de esta encuesta coinciden con aquellos reportados por Neilsen et al. en el sentido que ambos señalan la importancia de la información para la ejecución. Más allá de esto, Martin enfatiza la necesidad de una mayor participación de toda la organización en los temas de la estrategia.

Claramente compartir información sobre el negocio, su estrategia y los resultados con toda la organización es una práctica que refleja confianza en la gente. Este tipo de práctica gerencial y las creencias y valores subyacentes son característicos de las llamadas organizaciones de alto rendimiento. Por ejemplo, dentro del contexto de las prácticas primarias y secundarias identificadas por Neilson, la clarificación y comunicación de la estrategia a todos los niveles de la organización asume el convencimiento por parte de los líderes de que la gente tiene la capacidad de aportar y es importante para la ejecución. Similares comentarios podríamos hacer sobre las prácticas de administración del talento, el liderazgo y la innovación.

Esto quedará más claro en la siguiente sección donde resumimos las características de las organizaciones de alto rendimiento.

\section{Organizaciones de Alto Rendimiento}

La característica fundamental de las organizaciones de alto rendimiento es que son diseñadas para potenciar la capacidad de las personas como medio para lograr un desempeño superior en forma sostenible. En estas organizaciones existe la creencia central de que la gente es el activo más valioso de una empresa y, por lo tanto, los sistemas, procesos y políticas deben estar encaminados a facilitar el despliegue de la colaboración, el compromiso y la creatividad de todo el personal en la creación de valor para los stakeholders de la organización.

Las prácticas gerenciales y las consiguientes formas de trabajo en las organizaciones de alto rendimiento difieren significativamente de aquellas en las organizaciones donde predomina el estilo tradicional de mando y control, donde el poder, la información, la toma de decisiones y el control se concentran en la gerencia. Otros nombres asociados con las organizaciones de alto rendimiento incluyen la gerencia participativa, la gerencia basada en el empoderamiento o facultación y la gerencia basada en el compromiso. Lo particular de este estilo de gerencia es la confianza en la gente lo cual se despliega en un diseño de organizaciones con más libertad de ejecución y de sentido de propiedad sobre el trabajo realizado (ownership). Estas prácticas presentan un marcado contraste con la "gerencia basada en el control" que se caracteriza por prácticas conducentes al modelo de organización jerárquica tradicional regida por el estilo de mando y control.

El estudio sistemático de las organizaciones de alto rendimiento y el descubrimiento de sus bondades tiene su origen en experimentos realizados en los años cuarenta cuando se acuña el término de "gerencia participativa". De hecho, es en aquellos años cuando se inicia la disciplina de Desarrollo Organizacional cuyo propósito es el diagnóstico, análisis, diseño y mejoramiento de la efectividad 
Invest. pens. crit. (ISSN 1812-3864)

Vol. 6, No. 1, enero-abril 2018

pp. $65-80$

organizacional (French y Bell 1998). Dentro del contexto del desarrollo organizacional, una organización efectiva pudiera considerarse como sinónimo de una organización de alto rendimiento; sin embargo, para mí, el concepto de organización de alto rendimiento va más allá de la concentración en temas sociales (cultura, liderazgo, administración del talento) e incorpora temas tecnológicos como los sistemas de gestión y el uso de herramientas tecnológicas para potenciar las capacidades humanas.

¿Cuáles son las características de las organizaciones de alto rendimiento? De acuerdo al profesor Edward Lawler (1992 y 2001) de la Universidad del Sur de California, en las organizaciones de alto rendimiento, el sistema organizacional se caracteriza por los siguientes aspectos ampliamente difundidos en toda la organización:

- Información sobre la estrategia, procesos, resultados del negocio, eventos y retroalimentación de los clientes.

- Conocimientos sobre el sistema de trabajo y el negocio.

- Poder para actuar y tomar decisiones sobre el trabajo realizado.

- Recompensas alineadas a los resultados del negocio, al crecimiento y competencias individuales, y a la contribución.

Estas características emergen de un diseño organizacional que tiende a una estructura achatada y flexible, procesos autónomos y auto-contenidos, sistemas de gestión con objetivos y medidores alineados a la estrategia e información sobre los resultados ampliamente compartida, y prácticas gerenciales orientadas al desarrollo de competencias y el trabajo en equipo. Todo esto sólo es posible cuando existe una cultura de confianza en la gente que promueva la participación, la iniciativa, el trabajo en base a objetivos, la búsqueda de la excelencia y la colaboración.

\section{La Cultura Predominante en las Empresas Panameñas}

La cultura de una organización se define formalmente como "el patrón de supuestos compartidos que el grupo ha aprendido al resolver sus problemas de adaptación externa e integración interna, que ha sido suficientemente efectivo para considerarlo válido y por consiguiente, a ser enseñado a los nuevos miembros como la forma correcta de percibir, pensar y sentir en relación a esos problemas." (Schein 2004, p.12). Estas formas de percibir, pensar y sentir se manifiestan en normas de conductas y, a su vez, en comportamientos tales como estilos de mando, relaciones interpersonales, procesos para la toma de decisiones, trato con clientes, proveedores y otros grupos de interés externos a la organización, y en el diseño de estructuras de trabajo y políticas.

Generalmente, cuando hablamos de cultura, nos referimos a lo que se valora y no se valora en una organización y lo que se espera de la gente en cuanto a comportamientos. Este entendimiento es un aspecto o estado intermedio de la cultura, entre los supuestos compartidos (el origen) y las manifestaciones organizacionales (los resultados: relaciones, procesos, políticas, etc.). Típicamente, es este aspecto intermedio de la cultura el cual se mide, y existen varios modelos de medición para ello. 
Invest. pens. crit. (ISSN 1812-3864)

Vol. 6, No. 1, enero-abril 2018

pp. $65-80$

Sin embargo, estas mediciones no deben confundirse con la medición del clima laboral (grado de satisfacción de la gente con diversos aspectos de la empresa), algo que suele ocurrir en nuestro medio.

En esta sección presento los resultados de la medición de la cultura organizacional en diez empresas panameñas, siete privadas y tres gubernamentales. Estas investigaciones incluyeron cerca de 2000 encuestados. El instrumento de medición utilizado se basa en el modelo desarrollado por Cook y Lafferty (1989) de Human Synergistics International (HSI). En este instrumento se solicita información sobre la situación actual y la situación deseada, aquella que contribuiría eficazmente al mejor desempeño de la empresa y satisfacción del personal.

De acuerdo al modelo de HSI, la cultura de una organización puede ser descrita cuantitativamente en función de doce dimensiones, componentes o estilos que se agrupan, a su vez, en tres clases de culturas, a saber: culturas constructivas, culturas defensivas-pasivas y culturas defensivas-agresivas.

Las culturas constructivas se caracterizan por una orientación equilibrada hacia el desempeño y las personas. Este tipo de cultura refleja los rasgos de las culturas de alto rendimiento descritas en la sección anterior. En este tipo de cultura se cree que un alto rendimiento se logra a través de personas motivadas y capacitadas que trabajan en forma colaborativa en el logro de objetivos específicos. En cuanto a mecanismos de motivación, este tipo de cultura hace énfasis en las necesidades superiores de la jerarquía de Maslow (necesidades sociales o de pertenencia, de reconocimiento y de autorealización). El estilo característico de liderazgo en este tipo de cultura es el participativodesarrollador, el cual enfatiza el logro de metas, el desarrollo y facultación de las personas y asume que en general las personas son maduras, obran de buena voluntad y desean ser tratadas como adultas. En las culturas constructivas predominan los siguientes componentes (el número entre paréntesis indica la posición dentro de la gráfica circular del perfil propuesto por HSI):

$\checkmark$ Cultura de realización o logro

$\checkmark$ Cultura auto-actualizadora (calidad y crecimiento)

$\checkmark$ Cultura humanística (participativa y centrada en la gente)

$\checkmark$ Cultura afiliativa (apertura y sensibilidad humana)

Se espera de sus miembros:

$\checkmark$ Orientación hacia la acción y el logro de metas.

$\checkmark$ Responsabilidad por los resultados.

$\checkmark$ Actitud de aprendizaje e innovación.

$\checkmark$ Participación en la toma de decisiones y la solución de problemas.

$\checkmark$ Colaboración y trabajo en equipo.

$\checkmark$ Comunicaciones abiertas y auténticas.

$\checkmark$ Amabilidad y trato cálido con los demás.

Las culturas defensivas-pasivas, por su parte, se caracterizan por un énfasis en temas humanos como amoldarse a la cultura y costumbres de la empresa, dándole relativa poca importancia a las tareas y los resultados. Los mecanismos de motivación en estas culturas enfatizan las necesidades inferiores de la jerarquía de Maslow (necesidades básicas, necesidades de seguridad y las necesidades sociales). 
Invest. pens. crit. (ISSN 1812-3864)

Vol. 6, No. 1, enero-abril 2018

pp. $65-80$

El estilo de liderazgo tiende a ser complaciente, poco exigente en cuanto a resultados y paternalista. También suele darse el estilo burocrático el cual promueve el apego a las reglas establecidas aun por encima de consideraciones relativas al desempeño. En estas culturas no hay mucho incentivo por alcanzar metas desafiantes y ser innovador, se reprime el conflicto y se valora ajustarse al "status quo". Estas culturas resisten el cambio en forma sutil, de allí el nombre de "defensivas". Los componentes que predominan son:

$\checkmark$ Cultura de (búsqueda de) aprobación

$\checkmark$ Cultura convencional (tradicional, burocrática)

$\checkmark$ Cultura dependiente (de los jefes)

$\checkmark$ Cultura de evasión (de la responsabilidad y el riesgo)

Los miembros de esta cultura creen que:

$\checkmark$ Sus actuaciones no deben arriesgar su propia seguridad en el trabajo.

$\checkmark$ Su forma de pensar y actuar debe ajustarse a las normas de conducta de la empresa.

$\checkmark$ Deben evitar tomar iniciativas o decisiones lo cual es responsabilidad de los jefes.

$\checkmark$ Hay que quedar bien con todos, evitar los conflictos y nunca desafiar a un jefe. Deben evitar el riesgo y ser culpados por algún error.

Finalmente, las culturas defensivas-agresivas se caracterizan por una marcada orientación hacia los resultados y las tareas, con una baja consideración a los aspectos humanos de la organización. Los mecanismos de motivación en estas culturas enfatizan las necesidades inferiores de la jerarquía de Maslow así como las necesidades de reconocimiento. En estas culturas se espera un mando fuerte, predominando el estilo de liderazgo autócrata y, en menor grado, el estilo manipulador. Ambos estilos, sobre todo el autócrata, presentan una orientación paternalista, asumiendo que las personas son inmaduras y deben ser coaccionadas y controladas (estilo autócrata) o manipuladas (estilo manipulador) para que realicen su trabajo adecuadamente. Estas culturas se caracterizan por altas puntuaciones en los siguientes componentes:

$\checkmark$ Cultura antagónica (confrontacional, cuestionamiento)

$\checkmark$ Cultura de poder (poca participación)

$\checkmark$ Cultura competitiva (relaciones ganar-perder)

$\checkmark$ Cultura perfeccionista (hay que estar encima de todo)

Se espera de sus miembros que:

$\checkmark$ Critiquen, desafíen, cuestionen y culpen a los demás, manteniendo un alto nivel de competencia interna.

$\checkmark \quad$ Estén al tanto de los errores de otros.

$\checkmark$ Se centren en la realización de las tareas individuales o de sus áreas de trabajo, sin considerar el impacto en otras personas o áreas de trabajo.

$\checkmark$ Logren resultados por medio del control, las órdenes y el poder de la posición más que a través de la colaboración y el compromiso del personal. 
Invest. pens. crit. (ISSN 1812-3864)

Vol. 6, No. 1, enero-abril 2018

pp. $65-80$

En las culturas defensivas-agresivas se espera que las personas trabajen arduamente, aun a expensas de su tiempo personal y el ambiente de trabajo suele ser tenso. Debido al perfeccionismo, a la crítica y al señalamiento de culpables cuando las cosas salen mal, es común que exista un relativo alto grado de miedo a equivocarse o a las represalias por expresar ideas contrarias. Aunque en situaciones de crisis y de competencia muy agresiva esta cultura puede ser altamente efectiva, a mediano y largo plazo sus características tienden a inhibir la innovación y la capacidad para la adaptación. Asimismo, la competencia interna y el excesivo cuestionamiento dan lugar al efecto "silo" (poca coordinación entre diferentes unidades de la organización). Finalmente, altos niveles de los estilos antagónico, competitivo y de poder incrementan conductas asociadas a la cultura defensiva-pasiva, particularmente al estilo evasivo.

En la siguiente página aparece el perfil promedio de las 10 empresas encuestadas en cuanto a la situación actual y la deseada, aquella que según los encuestados produciría los mejores resultados.

Como se puede observar, la cultura promedio en el grupo de empresas analizadas es la cultura defensiva-agresiva. Esta cultura predominó en todas las empresas encuestadas. A nivel del promedio, el estilo antagónico muestra la mayor intensidad, llegando casi al percentil $90 \%$ de las miles de empresas que han realizado esta medición a nivel mundial. El segundo estilo es el competitivo, prácticamente empatado con el estilo evasivo de la cultura defensiva-pasiva. La intensidad del estilo evasivo era de esperarse dada la intensidad de los estilos antagónico y competitivo, y del estilo de liderazgo promotor de estos comportamientos. También llama la atención que aunque el estilo perfeccionista está a nivel del percentil 75\%, los encuestados perciben que un mayor perfeccionismo sería beneficioso para la empresa. Finalmente, advertimos los niveles relativamente bajos de los rasgos asociados a la cultura constructiva. Todos los estilos de esta cultura, exceptuando el auto-realizador, están por debajo de los estilos defensivos. Es interesante notar que el estilo auto-realizador está justamente en el percentil 50\% (mediana) de las empresas que han utilizado esta medición. Los demás estilos de la cultura constructiva muestran valores inferiores a la mediana.

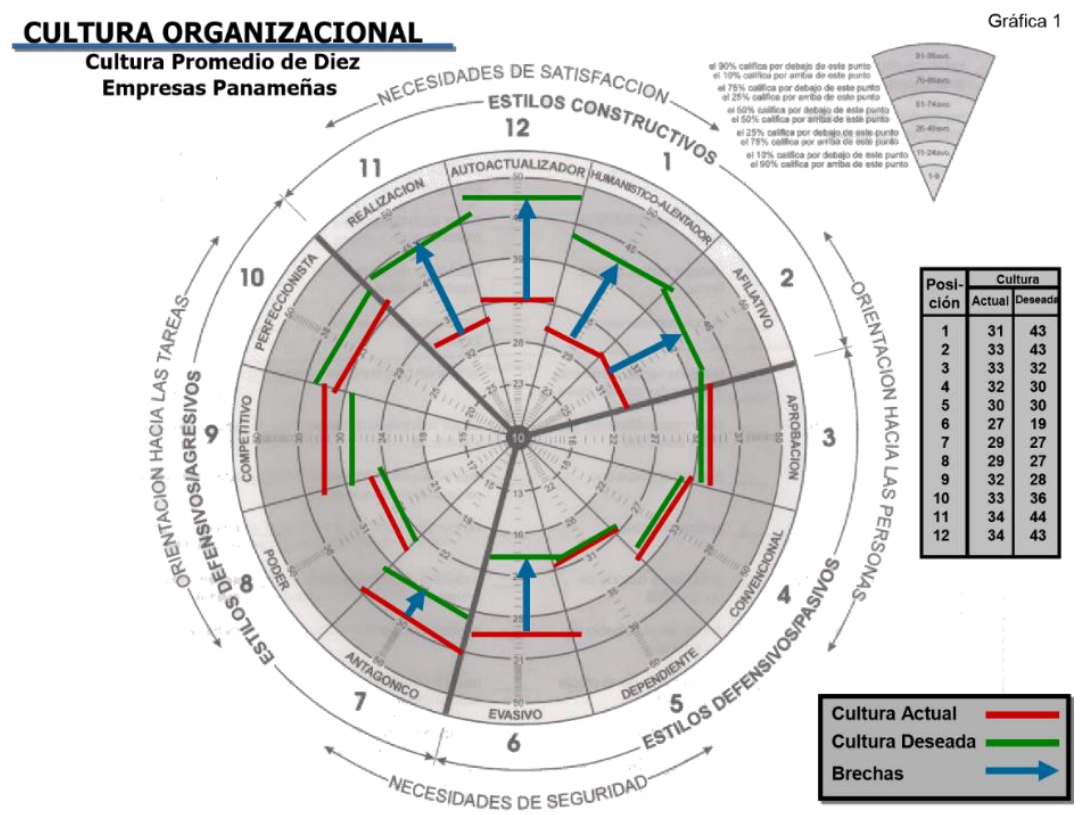


Invest. pens. crit. (ISSN 1812-3864)

Vol. 6, No. 1, enero-abril 2018

pp. $65-80$

En cuanto a la cultura deseada, aquella identificada como la más productiva, claramente los encuestados señalan la cultura constructiva. Esto es típico en todas las investigaciones, la gente intuitivamente identifica los comportamientos inductores del alto rendimiento. De hecho, en esta investigación, las menores dispersiones en las respuestas se dieron en los estilos constructivos. Además, es importante notar que es en la cultura constructiva donde se dan las mayores brechas a ser cerradas por las intervenciones de cambio organizacional.

¿Por qué predomina la cultura defensiva-agresiva en las empresas encuestadas aun cuando existe un conocimiento intuitivo de los rasgos inductores del alto rendimiento? Una explicación plausible es que la cultura nacional, aquella que desde niños influencia nuestra percepción de lo valorado y no valorado en cuanto a conductas, fomenta un estilo de liderazgo con mando fuerte como el estilo ideal de los gerentes. Ciertamente, este es un rasgo de las llamadas culturas

"masculinas", una de las cuatro dimensiones de la cultura nacional según el modelo del Prof. Geert Hofstead (2001), uno de los investigadores más reconocidos en el análisis de las culturas nacionales. Según este modelo las dimensiones que ayudan a descifrar la cultura de un país son:

1. Distancia de poder: Grado de aceptación de las desigualdades de poder en las organizaciones.

2. Aversión a la incertidumbre: Grado en que una sociedad se siente amenazada por la incertidumbre, el riesgo, la ambigüedad y las situaciones poco estructuradas.

3. Individualismo: Grado de preferencia por actuar como individuos vs. actuar como miembros de un grupo; es decir, valor que se le da a los objetivos personales, la autonomía y la privacidad por sobre la lealtad al grupo y el compromiso con las normas y actuaciones grupales.

4. Masculinidad: Grado en el cual la sociedad otorga mayor valor a rasgos "masculinos" como la riqueza, la ambición, el logro, la agresividad y la competencia vs. los rasgos de valoración "femeninos" entre ellos, la pertenencia, la igualdad, la consideración, ayudar a los demás y la preservación de la naturaleza.

Este modelo ha sido utilizado para explicar diferencias entre países en temas de negocios. Por ejemplo, Germaine-Grant et al. (2001) analizaron las diferencias en la toma de decisiones entre Australia y Singapur. Por otro lado, Hidalgo Campos et al. (2007) hacen un repaso de varios estudios sobre la cultura chilena y su impacto sobre la administración, el manejo de los recursos humanos y el mercadeo (comportamiento de los consumidores).

En ausencia de una medición de la cultura nacional para Panamá, mostramos a continuación una adaptación de las mediciones de Fernández et al. (1997), para cinco países de interés, tres de Latinoamérica, para aproximar a Panamá, y Alemania y Estados Unidos. 
Invest. pens. crit. (ISSN 1812-3864)

Vol. 6, No. 1, enero-abril 2018

pp. $65-80$

Valores de las Dimensiones de Hofstede para Cinco Países

Fuente: Fernández et al. (1997) citado en Hidalgo Campos et al. (2007)

\begin{tabular}{|l|l|l|l|l|}
\hline & $\begin{array}{l}\text { Distancia de } \\
\text { poder }\end{array}$ & $\begin{array}{l}\text { Aversión a la } \\
\text { incertidumbre }\end{array}$ & Individualismo & Masculinidad \\
\hline Chile & 12.71 & 14.73 & 12.17 & 12.10 \\
México & 14.15 & 12.31 & 12.23 & 12.54 \\
Venezuela & 12.15 & 14.08 & 11.83 & 10.49 \\
\hline Promedio & 13.00 & 13.71 & 12.08 & 11.71 \\
Alemania & 11.89 & 12.36 & 11.64 & 10.46 \\
EUA & 12.70 & 14.88 & 13.41 & 10.46 \\
\hline
\end{tabular}

Una revisión rápida de estas cifras indica que en sólo una de las dimensiones hay dominancia por parte de los tres países latinoamericanos frente a Alemania y EUA: la masculinidad. Los tres países muestran niveles superiores de valoración de los rasgos masculinos. Aunque estos datos están lejos de explicar formalmente la razón de la alta incidencia de la cultura defensiva-agresiva en Panamá, creo que los mismos representan una aproximación en la dirección correcta.

Posiblemente, más interesante que explicar el por qué de la incidencia de la cultura defensivaagresiva es tratar de identificar las consecuencias a nivel de la competitividad de las empresas y del país en general. En primer lugar, este tipo de cultura no conlleva a la máxima utilización del talento dentro de las organizaciones. Cuando mucho, trata de maximizar el aporte de la alta gerencia. En segundo lugar, la desconfianza en la gente inherente a esta cultura se trasluce en las prácticas gerenciales asociadas a la misma, lo cual desmotiva al personal, atentan contra su auto estima y, por ende, obstaculiza su desarrollo y cercena su potencial de aportar. Tercero, típicamente los cambios y las innovaciones introducidas por la alta gerencia serán resistidos por el resto de la organización en forma sutil, ya sea por miedo a perder relevancia como personas, por desconocimiento del alcance del cambio o simplemente por falta de confianza en la alta gerencia. En resumen, la estrategia no será el trabajo de cada uno en la organización y la competitividad estará por debajo de su máximo potencial.

\section{Conclusiones}

Las organizaciones de alto rendimiento reflejan altos niveles de adaptabilidad a las cambiantes circunstancias del entorno. Esta adaptabilidad se apoya en un entendimiento de la estrategia por parte de toda la organización, en la claridad de la asignación de responsabilidades y rendición de cuentas, en el alineamiento de objetivos e iniciativas con la estrategia, en información sobre el negocio y los resultados ampliamente compartida por todos en la organización, en el empoderamiento para la toma de decisiones, y en recompensas compartidas sobre los beneficios logrados. En su conjunto, estas características producen capacidad de ejecución.

La capacidad de ejecución emerge del sistema organizacional, cuyo componente central, la cultura, influye poderosamente sobre el comportamiento de las personas, y es, a fin de cuentas, a través de los comportamientos de las personas que se realizan las tareas asignadas a los humanos en la empresa. 
Invest. pens. crit. (ISSN 1812-3864)

Vol. 6, No. 1, enero-abril 2018

pp. $65-80$

De los tres estilos de cultura referidos, la cultura constructiva describe aquellos rasgos asociados con las organizaciones de alto rendimiento. No obstante, en las empresas panameñas estudiadas, fue justamente en la cultura constructiva, donde se observaron las mayores brechas a ser llenadas por una agenda de cambio apropiada.

Para lograr resultados superiores en el desempeño, la agenda de cambio debe estar guiada por objetivos de mejoras concretas en los resultados de negocios y de cambio en las prácticas gerenciales. Las iniciativas de mejoras típicamente propuestas por el área de recursos humanos (capacitación, coaching, etc.) no serán suficientes. Será necesario simplificar la estructura organizacional para mejorar la claridad de la asignación de responsabilidades, rediseñar los procesos de negocios claves y, sobre todo, fortalecer el sistema de gestión para traducir la estrategia en objetivos y métricas a todos los niveles de la organización y producir información

"accionable" para el seguimiento y mejoramiento del desempeño. Finalmente, los estilos de liderazgo y prácticas gerenciales deben facilitar la facultación y el desarrollo de la gente, primordialmente a través de la participación en la toma de decisiones. Esto producirá el sentido de propiedad (ownership) indispensable para asegurar la colaboración, el compromiso y la creatividad de todo el personal en la creación de valor para los stakeholders.

Así se cambiará la cultura, desarrollaremos a nuestra gente, aumentará la capacidad de ejecución, y se sentarán las bases para la competitividad y el desarrollo empresarial sostenible.

\section{Referencias}

1. Bossidy, L. y R. Charan, Execution: The Discipline to Get Things Done, Crown Business, 2002.

2. Cooke, R. y J. C. Lafferty, Organizational Culture Inventory, Human Synergistics, 1989.

3. Fernández, D., D. Carlson, L. Stepina, y J. Nicholson, "Hostede’s Country Classification, 25 years later", The Journal of Social Psychology, 137, 1997.

4. French, W. L. y C. H. Bell, Organizational Development, 6 ${ }^{\text {th }}$ edition, Prentice Hall, 1998.

5. Germaine-Grant, S., S. Petrovic-Lazarevic y M. Barrell, "Significance of Recognition of Australian and Singaporean Cross-Cultural Differences in the Decision-Making Process", International Journal of Management Literature, 1(1), 2001.

6. Hall, J., The Competence Connection: A Blue Print for Excellence, Woodstead Press, 1988.

7. Hidalgo Campos, P., E. Manzur Mobarec, S. Olavarrieta Soto y P. Farías Nazel, "La Cultura Nacional y su Impacto en los Negocios: El Caso de Chile”, Estudios Gerenciales, 23(105), 2007. Chile.

8. Hofstede, G., Culture's Consequences: Comparing Values, Behaviors, Institutions and Organizations across Nations, $2^{\text {nd }}$ edition, Sage Publications, 2001. 
Invest. pens. crit. (ISSN 1812-3864)

Vol. 6, No. 1, enero-abril 2018

pp. $65-80$

9. Hrebiniak, L., Making Strategy Work: Leading Effective Execution and Change, Wharton School Publishing, 2005.

10. Kaplan, R. S. y D. P. Norton, The Strategy-Focused Organization, Harvard Business Press, 2001.

11. Kaplan, R. S. y D. P. Norton, The Execution Premium: Linking Strategy to Operations for Competitive Advantage, Harvard Business Press, 2008.

12. Kotter, J. P. y J. L. Heskett, Corporate Culture and Performance, Free Press, 1992.

13. Lawler III, E. E., The Ultimate Advantage: Creating the High Involvement Organization, JosseyBass, 1992, p. 116.

14. Lawler III, E. E., Designing High Performance Organizations, Nota Técnica, Center for Effective Organizations, University of Southern California, 2001.

15. (http://unpan1.un.org/intradoc/groups/public/documents/UN/UNPAN000695.pdf)

16. Martin, R. L., “The Execution Trap”, Harvard Business Review, 88(7/8), 2010.

17. Neilson, G. L., K. L. Martin y E. Powers, “The Secrets of Successful Strategy Execution”, Harvard Business Review, 86(6), 2008.

18. Nohria, N., W. Joyce y R. Roberson, "What Really Works", Harvard Business Review, 81(7/8), 2003.

19. Pascale, R. y A. Athos, The Art of Japanese Management, Simon \& Schuster, 1981.

20. Peters, T y R. Waterman, In Search of Excellence, Harper \& Row, 1982.

21. Schein, E., Organizational Culture and Leadership, $3^{\text {rd }}$ edition, Jossey-Bass, 2004. 